\title{
Once-daily, single-tablet HIV PEP promises high adherence
}

HIV postexposure prophylaxis (PEP) with once-daily use of a single tablet containing emtricitabine, rilpivirine and tenofovir disoproxil fumarate in men who have sex with men (MSM) has adherence and completion rates of $>90 \%$, according to a new report in Clinical Infectious Diseases.

\section{The PEP regimen was completed by $92 \%$ of the participants... 77}

Most of the currently used PEP regimens consist of three drugs and require individuals to take tablets twice or three times daily, which can result in low adherence rates and suboptimal effectiveness in preventing HIV acquisition. Now, an open-label, singlearm study conducted in Melbourne and Sydney, Australia, examined adherence and safety of a PEP regimen consisting of a single tablet of co-formulated emtricitabine $200 \mathrm{mg}$, rilpivirine $25 \mathrm{mg}$, and tenofovir disoproxil fumarate
$300 \mathrm{mg}$ taken once daily. These reverse transcriptase inhibitors act before HIV integration into the human genome and are rapidly absorbed after administration (aiding in reaching therapeutic drug concentrations quickly), which might be important for the effectiveness of PEP.

The investigators enrolled $100 \mathrm{HIV}$ noninfected, healthy MSM who were eligible for PEP. Treatment was started at a mean $2 \mathrm{~h}$ after presentation and lasted for 28 days. The study participants reported for up to seven study visits over 12 weeks, which included study drug dispensation and blood sampling. Primary end points were the proportion of participants with premature PEP cessation (before day 28) or primary HIV infection by week 12 .

The PEP regimen was completed by $92 \%$ of the participants, with self-reported adherence of $98.5 \%$ in the 92 men who completed the 28-day follow-up period and $98.6 \%$ by pill count in the 78 participants who returned their pill bottles at day 28 . The eight premature therapy cessations were due to loss to follow-up (6), study burden (1) or adverse events (1). Overall, clinical adverse events occurred in 88 men-most commonly fatigue (34\%) and nausea (23\%) - with four participants experiencing a clinical adverse event of grade 3 or higher that was possibly attributable to the study drugs.

To further determine adherence, plasma levels of tenofovir and emtricitabine were measured within $48 \mathrm{~h}$ of the last dose at week 4 in 41 men. $88 \%, 10 \%$ and $2 \%$ of these men had tenofovir levels $>40 \mathrm{ng} / \mathrm{ml}$, $20-40 \mathrm{ng} / \mathrm{ml}$ and $<10 \mathrm{ng} / \mathrm{ml}$, respectively, corroborating the high adherence rates.

The study was not statistically powered to assess efficacy but the investigators report that none of the 70 men (76\%) who attended the final follow-up visit had acquired an HIV infection at week 12.

Clemens Thoma

Original article Foster, R. et al. Single-tablet emtricitabine-rilpivirine-tenofovir as HIV post-exposure prophylaxis in men who have sex with men. Clin. Infect. Dis. doi:10.1093/cid/civ511 